\title{
Unusual association of alveolar rhabdomyosarcoma with pancreatic metastasis: emerging role of PET-CT in tumor staging
}

\author{
Priyanka Jha • Andreas M. J. Frölich • \\ Beth McCarville • Oscar M. Navarro • Paul Babyn • \\ Robert Goldsby • Heike Daldrup-Link
}

Received: 17 September 2009 /Revised: 19 December 2009 / Accepted: 22 January 2010 /Published online: 24 February 2010

(C) The Author(s) 2010. This article is published with open access at Springerlink.com

\begin{abstract}
Background Pancreatic metastases in childhood cancer have been rarely reported in the radiology literature although ample evidence exists in pathology reports for its occurrence in patients with alveolar rhabdomyosarcomas (RMS).

Objective Assess the occurrence of pancreatic metastases in alveolar rhabdomyosarcomas, increase awareness of this association and reassess current staging protocols.

Materials and methods Three major oncology centers reviewed their records and imaging examinations. Patients' history and demographics, primary tumor site and histology,
\end{abstract}

P. Jha · A. M. J. Frölich · H. Daldrup-Link

Department of Radiology and Biomedical Imaging,

University of California, San Francisco Medical Center,

San Francisco, CA, USA

B. McCarville

Department of Radiological Sciences (MS 220),

St. Jude Children's Research Hospital,

Memphis, TN, USA

O. M. Navarro $\cdot$ P. Babyn

Department of Diagnostic Imaging, Hospital for Sick Children, University of Toronto,

Toronto, Canada

\section{R. Goldsby}

Department of Pediatrics, University of California,

San Francisco Medical Center,

San Francisco, CA, USA

\section{H. Daldrup-Link $(\bowtie)$}

Department of Radiology, University of California San Francisco, 505 Parnassus Ave.,

San Francisco, CA 94143-0628, USA

e-mail: daldrup@radiology.ucsf.edu presence of tumor recurrence, and presence and location of other metastases were reviewed.

Results Pancreatic metastases occurred in eight patients with alveolar RMS. Four of these presented at diagnosis and four with disease recurrence. In recurrent disease, the duration between the diagnosis of the primary tumor and pancreatic metastases varied from 8 months to 6 years (mean \pm SD: $2.38 \pm 2.49$ years). In all patients who received PET scans, pancreatic metastases showed a marked FDGuptake, but had variable detectability with CT. Pancreatic metastases were not associated with certain primary tumor locations or presence of other metastases, mandating an evaluation of the pancreas in all cases of alveolar rhabdomyosarcomas.

Conclusion Radiologists should be sensitized and actively evaluate the pancreas in patients with alveolar RMS. Optimizing CT and PET-CT protocols may increase the diagnostic yield.

Keywords Alveolar rhabdomyosarcoma - Metastasis . Pancreas $\cdot$ Pancreatic metastasis $\cdot$ PET $\cdot$ CT $\cdot$ Children

\section{Introduction}

Rhabdomyosarcoma (RMS) represents the most common soft-tissue sarcoma in children, with an incidence of 4.3 new cases per million children and adolescents younger than 20 years [1]. RMS originates from striated muscle cells or their mesenchymal precursors and occurs in a large variety of locations, such as the head and neck, pelvis and extremities [2]. Histologically, RMS can be classified as either embryonal $(60 \%)$, alveolar $(20 \%)$, undifferentiated or miscellaneous subtypes [3]. Alveolar RMS is associated 
with an aggressive course and a poor outcome [3]. Alveolar RMS frequently has genomic alterations such as chromosomal translocations with fusion of the FKHR gene on chromosome 13 with either PAX 3 (on chromosome 2) or PAX 7 (on chromosome 1) [4], which lead to chimeric fusion proteins that alter cellular transcription and amplify cell proliferation [4]. While multimodality treatment studies and specifically the use of radiation therapy have shown improved outcomes [5], the development of distant metastases still correlates with 5-year survival rates of less than $20 \%[3,6]$. Metastatic disease is thought to involve most commonly the lungs, bone, bone marrow and lymph nodes [6] and rhabdomyosarcomas have a propensity to involve unusual sites such as the breast, testes and subcutaneous tissue [7]. In addition, previous autopsy studies reported an incidence of pancreatic metastases in $67 \%$ of patients who died from metastatic alveolar RMS [7]. Conversely, only reports of isolated cases of patients with alveolar RMS and pancreatic metastases can be found in the radiology literature $[2,8-10]$. Here, we report a series of eight cases of metastatic involvement of the pancreas in patients with alveolar RMS. We want to sensitize radiologists to the possible association of pancreatic metastases in patients with alveolar RMS, which warrants careful and active evaluations of the pancreas in these patients and optimization of imaging protocols.

\section{Materials and methods}

The Committee on Human Research at three major pediatric oncology centers approved this retrospective evaluation. Pediatric radiologists at UCSF Children's Hospital, Hospital for Sick Children, which is affiliated with the University of Toronto, and St. Jude Children's Research Hospital reviewed their records of the last 11 years (1998-2009) and identified eight patients with biopsyproven alveolar RMS and pancreatic metastases out of a total of 71 cases (males:females $=34: 37$ ) of alveolar rhabdomyosarcoma. All clinical data and imaging studies of these patients were reviewed retrospectively, including patient's age, gender, clinical presentation, primary tumor location, presence or absence of metastases at diagnosis, type of therapy, presence or absence of recurrence after therapy, metastases at time of recurrence, duration between initial diagnosis and imaging findings of pancreatic metastases and final outcome.

The eight subjects included three males and five females, ages 1.8 to 35.0 years (mean \pm SD $8.0 \pm 4.4$ years). Interestingly, all younger patients were females while the males in this series presented with disease in the pubertal and postpubertal years. The characteristics of the patients are summarized in Table 1. The diagnosis of alveolar RMS was confirmed on histologic biopsy of the primary tumor. Medical history, treatment history and imaging studies were reviewed.

Evaluations of imaging studies included the following:

Imaging studies at diagnosis of the primary tumor revealed that all eight patients received an MR and three had a CT of the primary tumor. In addition, all patients received a contrast-enhanced chest $\mathrm{CT}$ (CECT) at diagnosis. Abdominal axial contrast-enhanced CT scans covered the pancreas in accordance to the Children's Oncology Group (COG) guidelines for initial staging in all the patients. Three patients received a PET and two received a PET-CT. Three patients didn't receive any PET examination.

The patients were followed with MRI of the primary tumor and CECT of the chest at weeks 6, 19, and 34 during therapy and at the end of therapy at week 54 per COG protocols. In addition, abdominal CECT was obtained in all patients at weeks $6,19,34$ and 54 except four instances, when non-enhanced abdominal CTs were obtained in two patients at weeks 6 and 34 of their respective follow-ups.

PET-CT studies were employed for four of these patients who were deemed to be high risk or for evaluating suspected widespread metastases for treatment planning. All imaging studies were retrospectively reviewed by two independent pediatric radiologists at each center concerning the presence, number, size, location and imaging characteristics of pancreatic metastases.

In acquiring the diagnostic-quality CTs associated with this study, the effective dose ranged between 1.76 and $24.7 \mathrm{mSv}$ for all patients. The radiopharmaceutical 18F-FDG was intravenously injected at a rate of $0.21 \mathrm{mCi} / \mathrm{kg}$ of body weight $(7.78 \mathrm{MBq} / \mathrm{kg})$ with a mean activity of $14.37 \mathrm{mCi}$ (531.69 MBq $/ \mathrm{kg}$ ) administered. Thus, based on the biodistribution of $18 \mathrm{~F}-\mathrm{FDG}$ and the ICRP internal dosimetry model, the radiation exposure of a patient who received the mean dose of $18 \mathrm{~F}-\mathrm{FDG}$ was $1.58 \mathrm{rem}(12.2 \mathrm{mSv}$; http://www. internaldosimetry.com; CDE Dosimetry Services, Inc. 2001). The combination of PET and CT provided a mean effective dose of $23.49 \mathrm{mSv}$ (range 19.12 to $55.06 \mathrm{mSv}$ ).

\section{Results}

The seven children included three boys and five girls, ages 1.8 to 20.0 years (mean \pm SD: $8.0 \pm 4.4$ years). In addition, an adult patient (35-year-old man) with alveolar RMS was identified. Clinical findings of these patients are summarized in Table 1. All patients had a biopsy-proven alveolar RMS at variable locations, in the upper extremity (4 cases), head and neck (2), lower extremity (1) and pelvis (1). The chemotherapy regimens instituted included the following protocols: (1) POGD-9803, regimen B with vincristine, actinomycin and cyclophosphamide; (2) Irinotectan along 


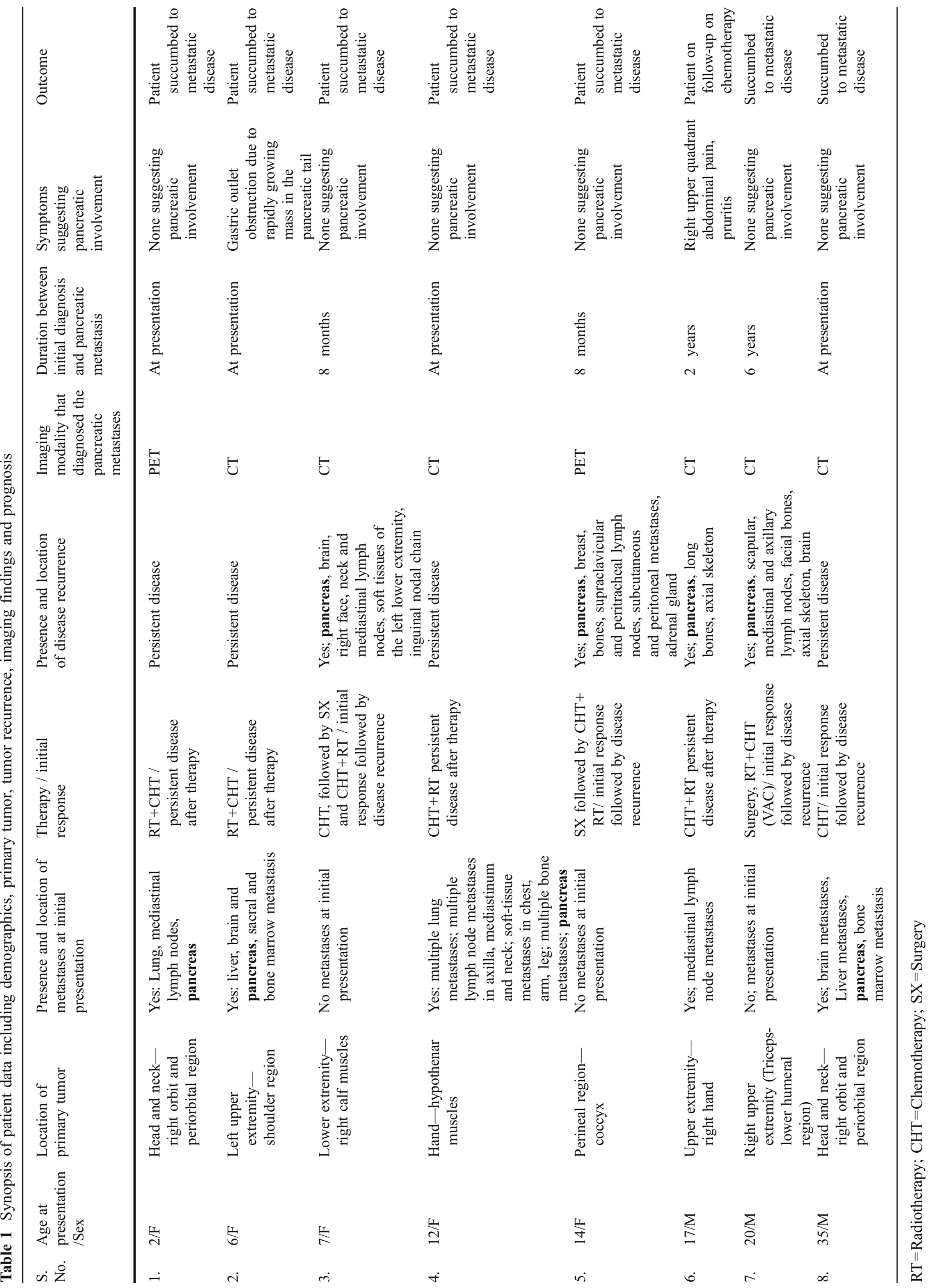



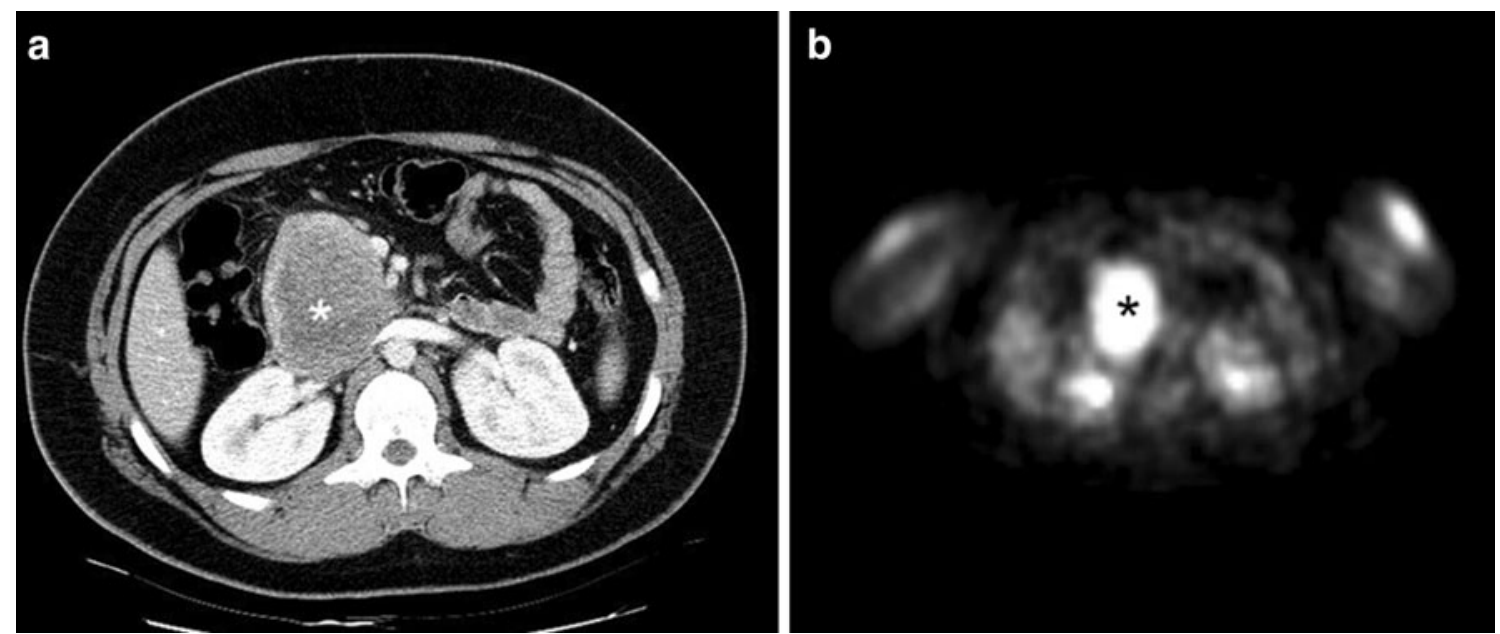

Fig. 1 A 17-year-old boy with previously treated alveolar RMS of the right hand presented with increasing right upper quadrant abdominal pain and pruritus. a Axial CECT scan demonstrates a large, hypodense

with radiotherapy and autologous bone marrow transplantation; (3) D9802, regimen B (vincristine, actinomycin and cyclophosphamide, regimen B). The common drugs that were used in all patients were vincrisitine, actinomycin and cyclophosphamide.

Four patients had pancreatic metastases diagnosed at initial presentation while four patients presented with pancreatic metastases at the time of disease recurrence. The age at diagnosis of the pancreatic metastases varied from 2 years to 35 years (mean \pm SD: $15.38 \pm 10.98$ ). The duration between the diagnosis of the primary tumor and that of pancreatic metastases in patients with recurrent disease varied from 8 months to 6 years (mean \pm SD: $2.38 \pm$ 2.49 years). In this small cohort of patients, the pancreatic metastases were observed with no relation to a specific primary tumor site. Six of our patients had upper extremity, mass $(*)$ in the head of the pancreas. The mass caused symptoms of obstructive jaundice. b PET scan at the same level depicts marked radiotracer uptake (asterisk) of this lesion

head and neck primary disease and we note current COG recommendations regarding abdominal CT scans are only for lower extremity, genitourinary and abdominal tumors. Six pancreatic metastasis were clinically asymptomatic with the diagnosis being established on routine CT or PET staging or re-staging examinations. Two patients were symptomatic and presented with obstructive jaundice in one case (Fig. 1) and gastric outlet obstruction in the other (Fig. 2).

All CT imaging studies revealed metastases in the pancreas as irregular, hypodense lesions compared to surrounding enhancing pancreatic tissue on venous phase post-contrast scans (Figs. 1-4). Pancreatic metastases presented as solitary lesions in six patients (Figs. 1-4), two focal lesions in one patient and multiple lesions geographically involving the pancreas in one patient. The lesions were all well defined $(n=8)$ and located in the head (four lesions), body (4) or tail



Fig. 2 A 6-year-old girl with locally recurrent and metastastic alveolar RMS of the left upper extremity. a Axial CECT scan of the abdomen shows a large, irregular, inhomogeneous hypodense lesion

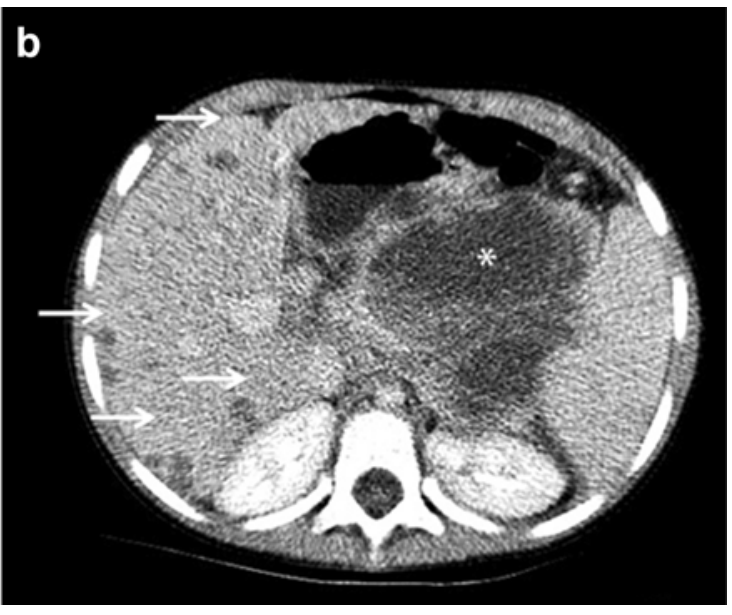

$\left(^{*}\right)$ in the tail of the pancreas. b Axial CT scan obtained 5 weeks later demonstrates marked interval growth of the lesion and development of multiple new intrahepatic lesions (arrows) 

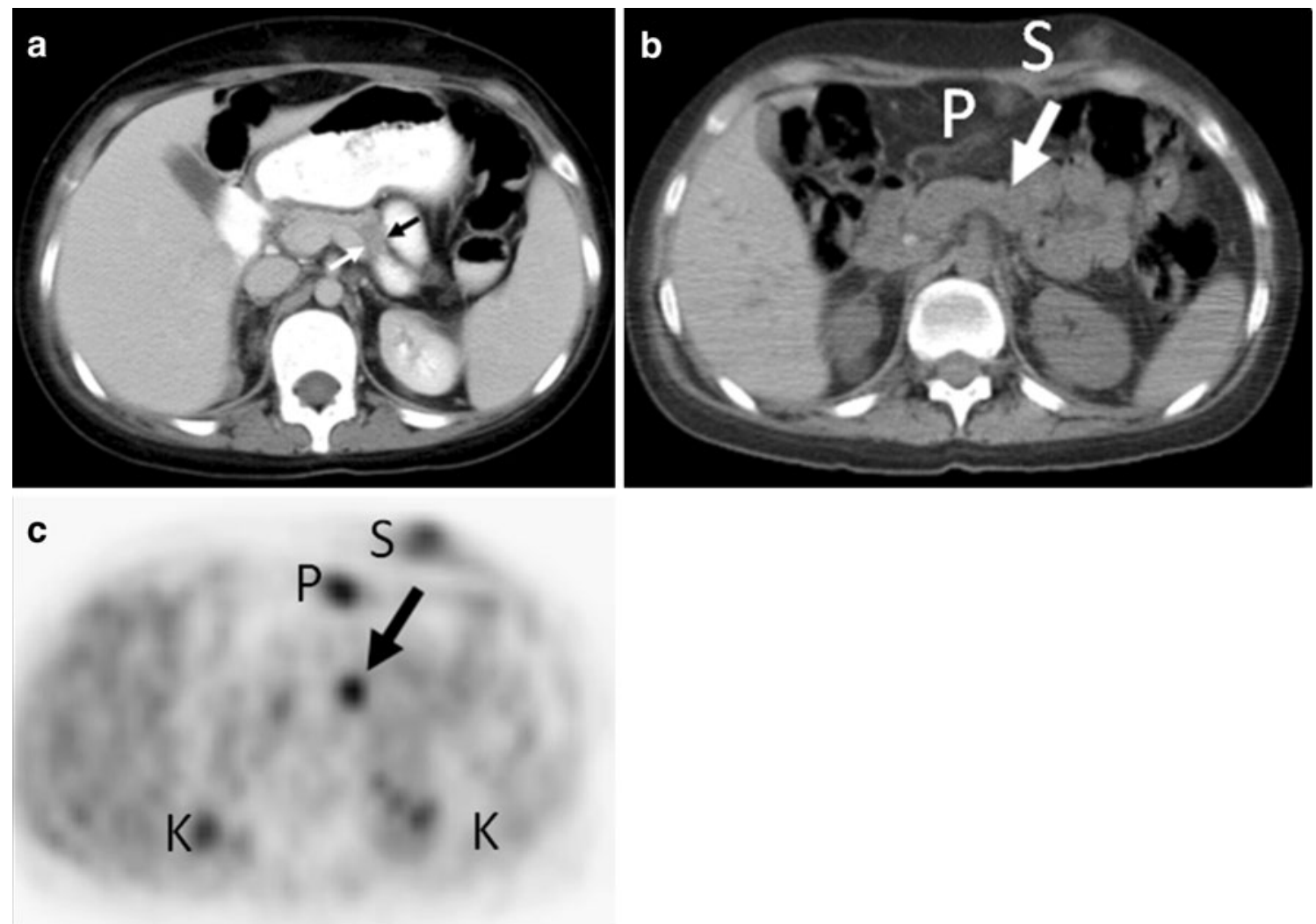

Fig. 3 A 14-year-old girl with alveolar RMS of the pelvis and pancreatic metastases. a Axial contrast-enhanced abdominal CT image demonstrates a small, hypodense mass in the body of the pancreas, which was initially missed on CECTs and detected on PET-CT imaging (arrows). b Axial CT obtained during PET-CT shows the area of the pancreas metastasis (arrow) and subcutaneous $(S)$ and

peritoneal metastases $(P)$. c Co-registered axial PET image, at the same level shown in (b), demonstrates small, intensely FDG-avid pancreas (arrow) and subcutaneous $(S)$ and peritoneal $(P)$ metastases. Two foci of FDG activity in the retroperitoneum correspond to normal kidneys $(K)$

(2) of the pancreas. One patient showed an associated intraand extrahepatic bile duct dilation (Fig. 1). PET scans were performed in three patients (Fig. 3) and an integrated PETCT scan (Figs. 1 and 4) was obtained in two. All pancreatic metastases evaluated with PET showed significant FDGuptake in the region of the pancreas. Two of these metastases showed a rim-enhancement, without any central

enhancement on CT but marked central FDG-uptake on PET scans (Fig. 4). Our data showed that one pancreatic metastasis in our cohort was difficult to detect on contrastenhanced CT as it presented as an ill-defined distortion in the shape of the tail of the pancreas rather than a discrete mass or a nodule (Fig. 3). This and one other metastasis were initially missed on non-contrast enhanced CT, while
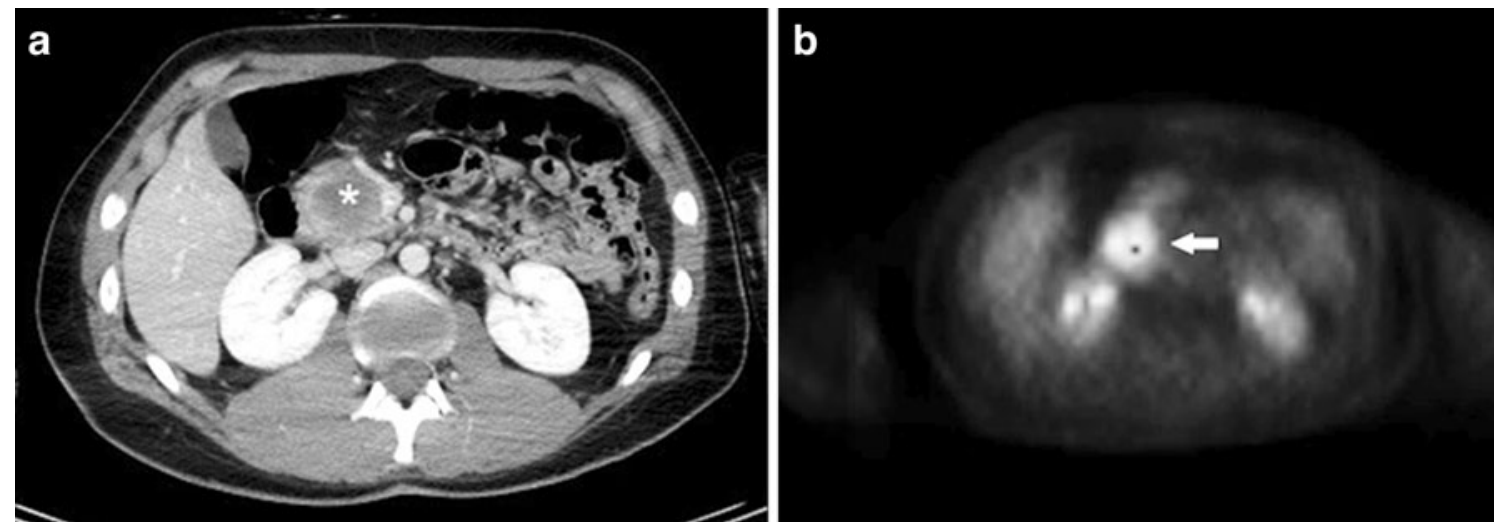

Fig. 4 A 20-year-old man with recurrent alveolar RMS of the right arm with widespread metastases. a Axial CECT scan demonstrates a low attenuation mass $(*)$ in the head of the pancreas. b PET scan at the level of the same lesion shows marked FDG uptake (arrow) 
PET or PET-CT easily diagnosed all pancreas metastases as hot spots.

In all patients, pancreatic metastases were diagnosed together with other metastases at extra-abdominal sites, such as lymph nodes $(n=6)$, bony metastases $(n=6)$, lung $(n=2)$, brain $(n=3)$, bone marrow $(n=1)$, breast $(n=1)$ or soft tissue $(n=4)$. Four patients had pancreatic metastases without any other metastases in the abdomen. The remaining four patients had pancreatic involvement with additional abdominal metastases in the liver $(n=2)$, adrenal glands $(n=1)$ or lymph nodes $(n=2)$. These additional intrabdominal metastases were diagnosed at the same time as the pancreatic metastases. None of the patients had isolated metastases in the pancreas without any metastatic involvement elsewhere in the body.

The outcome was dismal in seven of these patients who succumbed to widespread metastatic disease. The pancreatic metastases, however, can't be attributed to as an independent poor prognostic factor as it always occurred in the setting of widespread metastatic disease. One patient is currently being followed up on chemotherapy but also has a poor prognosis due to widespread metastatic involvement.

\section{Discussion}

Our retrospective study reveals an unexpected occurrence of pancreatic metastases in patients with alveolar RMS. The pancreas has been traditionally regarded as a rare site for metastases in children with cancer. However, according to an autopsy report of 57 children who died from alveolar RMS, 67\% had pancreatic metastases, making the pancreas the third most common site for metastases in these patients after lung and lymph nodes [7]. In spite of this high number, the presence of pancreatic metastases in RMS has been rarely reported in the radiology literature $[2,8,11$, 12]. This discrepancy between pathology and radiology observations may be due to different evaluated patient populations (selected patients with fatal outcome in the cited pathology report versus all patients with RMS evaluated on imaging studies), limited evaluations of the pancreas with standard imaging protocols and limited sensitivity of unenhanced CTs for the detection of pancreatic metastases among other parenchymal lesions.

There was no apparent correlation between the location of the primary tumor and the size and location of metastases in our small cohort. This is contrary to the usual thought that such metastases are usually expected with lower extremity and genitourinary tumors. According to the guidelines issued by the $\mathrm{COG}$, the initial staging examinations for soft-tissue sarcomas include a CT or MR scan for evaluating the liver and retroperitoneum. PET or PET-CT examinations are not included. It should also be noted that National Cancer Institute
(NCI) does identify that PET can help detect metastases otherwise undetected on conventional imaging and can be considered as a tool although not recommended by the current treatment protocols (http:/www.cancer.gov/cancertopics/pdq/ treatment/childrhabdomyosarcoma/HealthProfessional/ page4\#Reference4.2). Our data showed that two pancreatic metastasis in our study cohort were missed initially on CT, while PET or PET-CT easily diagnosed all pancreas metastases as hot spots due to their highly increased metabolic activity compared to the normal pancreas. Other authors also reported that they initially overlooked unsuspected pancreatic metastases in patients with RMS and that these were readily diagnosed on PET scans [8]. Also as detailed in the results section, pancreatic metastases may have only rim enhancement on CT studies but these same metastases avidly uptake FDG on PET imaging. This may be secondary to an extremely cellular center, which is underperfused leading to the discrepancy in CT and PET findings. Relying on CT alone may lead to a misdiagnosis of central necrosis and can be adjudged as a response to chemotherapy in an otherwise hypercellular mass. Several recent studies have suggested that the addition of PET to conventional imaging (chest, abdominal and pelvic CT, bone scan) may increase sensitivity for the detection of distant metastasis and lead to higher diagnostic accuracy for RMS staging [11-13]. Thus, inclusion of the upper abdomen on chest CT staging evaluations of RMS patients, adjustments of abdominal CT protocols toward better evaluation of the pancreas or inclusion of PET/CT studies into staging evaluations of patients with alveolar RMS should be considered to improve the detection of these lesions.

This increased incidence of unusual metastases, including pancreatic ones, has been noted by numerous authors in pathology literature [1, 4, 5, 14-16]. It has been postulated that RMS arising in the extremities, especially in the hand and foot, can be particularly aggressive with multiple metastases to unusual sites (e.g., breast, ovary, testes, kidney, pancreas) that most commonly have alveolar histology and, hence, worse prognosis [16]. The involvement of the pancreas even in the absence of other abdominal metastases, as seen in some of the cases presented here, raises the possibility that there might be an underlying biological cause for this pattern of metastatic spread. To the best of our knowledge, all previously described cases of pancreatic metastases in RMS were seen in tumors with alveolar histology [7, 9, 10, 16]. IGF-II receptor expression has been demonstrated on RMS cell lines [17-19]. It has also been reported that the PAX/FKHR fusion protein characteristic of alveolar RMS may induce transcription of IGF-I and other growth factors, further suggesting autocrine mechanisms of cellular proliferation $[18,19]$. This observation may be related to the known molecular alterations that are specific to alveolar RMS, such as the PAX3/7-FKHR fusion gene. 
The pancreas is also heavily influenced by IGF and IGF-like proteins. Such genetic associations also raise the possibility that the pancreas may be a part of multiorgan tumor involvement in susceptible tissues. This is further strengthened by the therapeutic effect of IGF-IR monoclonal antibodies on soft tissue sarcoma, including RMS. Future research should be directed towards better documenting and eliciting this curious relationship.

\section{Conclusion}

Our retrospective review suggests that pancreatic metastases in patients with alveolar RMS may be more common than previously reported. With this review, we intend to sensitize pediatric radiologists to evaluate imaging studies in these patients actively for pancreatic metastases. Further prospective studies have to show if addition of PET-CT evaluations will increase the sensitivity of standard imaging procedures and ultimately improve outcome by earlier detection of metastatic disease or disease recurrence.

Open Access This article is distributed under the terms of the Creative Commons Attribution Noncommercial License which permits any noncommercial use, distribution, and reproduction in any medium, provided the original author(s) and source are credited.

\section{References}

1. Sultan I, Qaddoumi I, Yaser S et al (2009) Comparing adult and pediatric rhabdomyosarcoma in the surveillance, epidemiology and end results program, 1973 to 2005: an analysis of 2, 600 patients. J Clin Oncol 27:3391-3397

2. McCarville MB, Spunt SL, Pappo AS (2001) Rhabdomyosarcoma in pediatric patients: the good, the bad, and the unusual. AJR 176:1563-1569
3. Pappo AS, Shapiro DN, Crist WM (1997) Rhabdomyosarcoma. Biology and treatment. Pediatr Clin North Am 44:953-972

4. McDowell HP (2003) Update on childhood rhabdomyosarcoma. Arch Dis Child 88:354-357

5. Womer RB, Pressey JG (2000) Rhabdomyosarcoma and soft tissue sarcoma in childhood. Curr Opin Oncol 12:337-344

6. Dagher R, Helman L (1999) Rhabdomyosarcoma: an overview. Oncologist 4:34-44

7. Enzinger FM, Shiraki M (1969) Alveolar rhabdomyosarcoma. An analysis of 110 cases. Cancer 24:18-31

8. McCarville MB, Christie R, Daw NC et al (2005) PET/CT in the evaluation of childhood sarcomas. AJR 184:1293-1304

9. Khalbuss WE, Gherson J, Zaman M (1999) Pancreatic metastasis of cardiac rhabdomyosarcoma diagnosed by fine needle aspiration. A case report. Acta Cytol 43:447-451

10. Farah RA, Kamen BA (1999) Parameningeal alveolar rhabdomyosarcoma with an isolated pancreatic metastasis. Pediatr Hematol Oncol 16:463-467

11. Tateishi U, Hosono A, Makimoto A et al (2009) Comparative study of FDG PET/CT and conventional imaging in the staging of rhabdomyosarcoma. Ann Nucl Med 23:155-161

12. Volker T, Denecke T, Steffen I et al (2007) Positron emission tomography for staging of pediatric sarcoma patients: results of a prospective multicenter trial. J Clin Oncol 25:54355441

13. Kleis M, Daldrup-Link H, Matthay K et al (2009) Diagnostic value of PET/CT for the staging and restaging of pediatric tumors. Eur J Nucl Med Mol Imaging 36:23-36

14. Loeb DM, Thornton K, Shokek O (2008) Pediatric soft tissue sarcomas. Surg Clin North Am 88:615-627, vii

15. DeWitt J, Jowell P, Leblanc J et al (2005) EUS-guided FNA of pancreatic metastases: a multicenter experience. Gastrointest Endosc 61:689-696

16. Miller DV, Coffin CM, Zhou H (2004) Rhabdomyosarcoma arising in the hand or foot: a clinicopathologic analysis. Pediatr Dev Pathol 7:361-369

17. van Golen CM, Schwab TS, Kim B et al (2004) Insulin-like growth factor-I receptor expression regulates neuroblastoma metastasis to bone. Cancer Res 45:5193

18. El-Badry OM, Minniti C, Kohn EC et al (1990) Insulin-like growth factor II acts as an autocrine growth and motility factor in human rhabdomyosarcoma tumors. Cell Growth Differ 1:325-331

19. Ayalon D, Glasser T, Werner H (2001) Transcriptional regulation of IGF-I receptor gene expression by the PAX3-FKHR oncoprotein. Growth Horm IGF Res 11:289-297 